\title{
Germanica
}

35 | 2004

La figure de l'imposteur dans la littérature de langue allemande au $\mathrm{xx}^{\circ}$ siècle

\section{Die Begegnung der Hochstapler oder : von der Vertracktheit der Aggression Robert Neumanns Olympia als Parodie auf Thomas Manns Bekenntnisse des Hochstaplers Felix Krull}

La rencontre des imposteurs ou : De la complication de l'agression. Olympia de Robert Neumann, parodie de l'ouvrage Les Confessions du chevalier d'industrie Felix Krull de Thomas Mann.

\section{Holger Pils}

\section{OpenEdition}

\section{Journals}

Édition électronique

URL : http://journals.openedition.org/germanica/1788

DOI : $10.4000 /$ germanica. 1788

ISSN : 2107-0784

Éditeur

Université de Lille

Édition imprimée

Date de publication : 31 décembre 2004

Pagination : 91-104

ISBN : 9782913857148

ISSN : 0984-2632

Référence électronique

Holger Pils, «Die Begegnung der Hochstapler oder : von der Vertracktheit der Aggression

Robert Neumanns Olympia als Parodie auf Thomas Manns Bekenntnisse des Hochstaplers Felix Krull », Germanica [Online], 35 | 2004, Online erschienen am: 05 Oktober 2012, abgerufen am 06 Oktober 2020. URL : http://journals.openedition.org/germanica/1788; DOI : https://doi.org/10.4000/germanica. 1788

Ce document a été généré automatiquement le 6 octobre 2020.

(c) Tous droits réservés 


\section{Die Begegnung der Hochstapler oder : von der Vertracktheit der}

\section{Aggression \\ Robert Neumanns Olympia als \\ Parodie auf Thomas Manns}

Bekenntnisse des Hochstaplers Felix Krull $^{1}$

La rencontre des imposteurs ou : De la complication de l'agression. Olympia de Robert Neumann, parodie de l'ouvrage Les Confessions du chevalier d'industrie Felix Krull de Thomas Mann.

Holger Pils

In Olympia parodiert Robert Neumann den Thomas Mann und den Krull, die Olympia und den Robert Neumann und übertreibt alles noch auf recht komische Weise.

Hermann Kesten ${ }^{2}$

Schließlich gibt es noch eine Möglichkeit : Der große Parodist Neumann wollte eine KrullParodie schreiben. [...] Natürlich mußte dieser Versuch mißlingen. Marcel Reich-Ranicki ${ }^{3}$

1927 sandte der noch weitgehend unbekannte Robert Neumann seinen ersten Parodienband Mit fremden Federn an Thomas Mann. Das Dankschreiben zeugt von Wohlwollen: «Ich habe sogar im Familienkreis daraus vorgelesen, und wir haben 
Tränen gelacht. [...] Nach allgemeinem Urteil bin ich selbst sehr gut getroffen und darf es mir wohl zur Ehre rechnen, zweimal vertreten zu sein ${ }^{4}$.»

Weniger wohlwollend fiel Erika Manns Reaktion 1961 aus, als Neumanns Verleger Kurt Desch in einem Brief beiläufig erwähnte, Neumann habe «ein bezauberndes Buch geschrieben », das Olympia heiße und Thomas Mann gewidmet sei.

In diesem heiteren und überraschenden Buch erzählt Olympia als die Schwester von Felix Krull ihre Lebenserinnerungen. Das Schlusskapitel ist dem alternden Felix Krull gewidmet. Ich hoffe, sie werden an diesem Buch ihre helle Freude haben ${ }^{5}$.

Statt « heller Freude » zeitigte die Ankündigung im Hause Mann nur helle Aufregung. Die Mann-Erben gingen mit dem S. Fischer Verlag zunächst gegen die Werbung für den Roman, die ihn als "eine Art Gegenstück zum ,Felix Krull' [...], Ergänzung und lächelnde Fortsetzung » ankündigte ${ }^{6}$, vor und weiteten später die Klage gegen das Buch aus : Sie lautete auf Plagiat. Die Auseinandersetzung konnte erst im März 1962 beendet werden, ohne dass die zweite Klage streitig verhandelt worden war. Man verglich sich, nachdem Neumann geringfügige Änderungen vorgenommen hatte. Dennoch : Olympia bleibt eine Parodie auf die Bekenntnisse des Hochstaplers Felix Krull. Vielfältige Bezüge verweisen jedoch nicht ausschließlich in stilparodistischer Absicht auf den Roman Thomas Manns - mit der Hochstapelei greift Neumann vielmehr ein zentrales Motiv seines eigenen Werkes wieder auf. Darüber hinaus handelt es sich bei Olympia um einen Fall instrumentalkritischer Parodie: Neumann nannte das Buch später eine « hintergründige Ovation» und sprach von der "Vertracktheit der Aggression», die ihm innewohne ${ }^{7}$. Im Folgenden soll die «Hintergründigkeit» der Parodie, deren Gegenstand auch Thomas Mann selbst sowie Neumanns Beziehung zu ihm ist, erläutert werden. Daher sei auf diese Beziehung kurz eingegangen, bevor die Bezüge des Romans im Einzelnen aufgezeig werden.

Nach eigenen Aussagen gehörte die Lektüre der Werke Thomas Manns zu den prägenden literarischen Erlebnissen in Neumanns Jugend ${ }^{8}$. Er sei fasziniert gewesen von der «Meisterlichkeit» der Form, die zu angestrengter Nachahmung rief. Die Resultate seien jedoch nur langweilig und steril gewesen. In der Erbitterung darüber, dass sie außerdem schlicht übersehen wurden, sah Neumann den Antrieb, zu parodieren statt $\mathrm{zu}$ imitieren. Er entdeckte die Gabe, "andere Personen $\mathrm{zu}$ 'impersonisieren' - [...] eine hochstaplerische Begabung im Grunde »'. Die Parodien sollten Neumanns schriftstellerischen Erfolg begründen. Der eingangs zitierte Brief Thomas Manns an Neumann vermittelt - bei aller gewohnten Höflichkeit - den Eindruck, dass auch ihm die Parodien gefallen haben.

Später sollte Neumann die einseitige Reduzierung auf den Parodisten, die den eigenen Stil - so facettenreich und schwer wahrnehmbar er war - nicht würdigte, zu schaffen machen ${ }^{10}$. Tagebucheinträge Thomas Manns, in denen nichtparodistische Werke Neumanns erwähnt werden, zeigen keinerlei Leseeindrücke ${ }^{11}$. Thomas Mann bildete also keine Ausnahme - und er machte zudem bereits in dem ersten, oben zitierten Brief auf die Schwäche aufmerksam, die Neumann selbst seinen Thomas Mann-Parodien anhaften sah : Neumann parodiert mit ihnen Literatur, die selbst bereits parodistische Züge aufweist. 
6 Während des Exils besuchte Neumann Thomas Mann in Küsnacht, ohne dass es zu einer wirklich freundschaftlichen Annäherung gekommen wäre ${ }^{12}$. Die von Robert Neumann im Zusammenhang mit dem Prozess um Olympia vorgelegten Briefe aus dem Jahre 1955 sprechen ihre eigene Sprache. Wieder ging es um die Parodie :

Lieber, sehr verehrter Herr Robert Neumann,

es grenzt ans Skandalöse, daß ich Ihnen noch nicht für die freundliche Zueignung Ihres neuen Parodienbuches gedankt habe. [...] Aber ich habe wieder gestaunt über die Genialität komischer Einfühlung, die sich in der neuen Sammlung offenbart. Es gibt dergleichen nicht zum zweitenmal und hat es wahrscheinlich nie gegeben. Eine Art von mimischer Kritik ist das, die ich mir als Produkt der Reizbarkeit erkläre und also des Leidens, - sooft sie bloß als Lustigmacherei aufgefaßt werden mag. Und großartig ist, wie ein paarmal die Parodie aufgegeben wird, weil nichts mehr zu parodieren ist, und weil das Unglaubwürdig-Wirkliche im parodistischen Rahmen in scheußlicher Nacktheit erscheint. Eine stärkere Bloßstellung gibt es nicht. All Ihr Spott wirkt harmlos dagegen, und wer wollte nicht lieber verspottet als so zitiert $\operatorname{sein}^{13}$.

7 Teil des « Leidens », das Thomas Mann benannte, war auch ein Leiden an Thomas Mann selbst : Neumann fasste seine Thomas Mann-Parodien als unzulänglich auf ; Reizbarkeit und Leiden konnten dort nicht gemildert werden, wo es nichts zu parodieren gab, oder die Parodie, als Lustigmacherei verstanden, nur gegen das «Original» abzufallen schien - so sah es Neumann zumindest selbst :

Verehrter Herr Thomas Mann,

wie gütig von Ihnen, es so zu sehen. Ich selbst komme mir mitunter mit dieser singulären Fertigkeit wie der Mann vor, der ein Salonlöwe wird, weil er mit den Ohren wackeln kann. Nehmen Sie nur die Parodien auf Thomas Mann, die immer wieder ,im ersten Satze' stecken bleiben, und das nicht nur äußerlich : der Substanz können sie überhaupt nichts anhaben (wie ja überhaupt das Ausgezeichnete ebensowenig parodierbar ist, wie das Miserable) - ganz abgesehen davon, daß Sie je selbst der subtilste Thomas-Mann-Parodist sind und Ihre Meisterlichkeit immer wieder lächelnd in Anführungszeichen setzen. Bleibt für mich nur der Taschenspielertrick, Ihre Selbstparodie zu vergröbern und die Lacher so auf meine Seite zu bringen. Es sind keine guten Lacher ${ }^{14}$.

Thomas Mann konnte augenzwinkernd die "Meisterschaft» seines « hochstaplerischen » Künstlertums kommentieren, wie in den Bekenntnissen, und sie gleichzeitig ernst nehmen - und er wurde ernst genommen. Neumann parodierte und wurde, wie es ihm selbst schien, nicht recht ernst genommen; er blieb der «Salonlöwe », der "mit den Ohren wackeln» kann und «keine guten Lacher» provoziert. In der Jugend war es die stilistische " Meisterlichkeit » Thomas Manns, die sich nicht imitieren ließ, im Alter war es dazu die "Meisterlichkeit» des gelebten Lebens, die Neumann zu schaffen machte : "man », das heißt Neumann, « hatte es mit diesem Mann nicht leicht », schrieb er in seiner Autobiographie ${ }^{15}$. Er glaubte Thomas Manns « verschmitztes Doppelspiel » durchschaut zu haben: « ein goethisches Leben zu führen - Kustos seiner selbst, eines Thomas-Mann-Museums ; und zugleich Parodist seines eigenen Kustodentums ; und doch wiederzugleich höchst goethisch ,Respekt' verlangend». Thomas Mann habe erwartet, dass man «jenes mitwisserische Augenblinzeln, das er einem anbot, submissest zu übersehen hatte. »

9 Submission, Respekt und Übersehen war nun Neumanns Sache nicht: seine Parodien waren, wie Thomas Mann bemerkte, nicht einfach "Spaßmacherei », sondern sie stellten bloß, insbesondere dort, wo er glaubte Literatur nehme sich zu ernst. Dieser 
Vorwurf sollte in der Auseinandersetzung um Olympia - nach Thomas Manns Tod - vor allem Erika Mann treffen, die "Tochter des Nationalheiligen " ${ }^{16}$.

Rudolf Walter Leonhardt bemerkte, Neumann sei "gebannt von der Größe eines anderen", noch nicht "Gebrochenen" gewesen und meinte damit Thomas Mann ${ }^{17}$. Nach Thomas Manns Tod versuchte Neumann diesen Bann zu brechen, in Olympia noch hintergründig, doch bereits ohne sich auf stilparodistische Mittel allein zu beschränken - im Plagiatsstreit mit der « Entourage des Olympiers ${ }^{18}$ dann ganz offen.

In Neumanns Roman gibt sich Olympia de Croulle als Schwester Felix Krulls aus; ihre Memoiren machen einen Großteil des Buches aus. Die Perspektive ist wechselnd: Neben der Titelheldin kommen auch deren Tochter sowie « Felix Krull » zu Wort. Olympia erläutert dem Leser, d.h. dem Leser der Bekenntnisse, ihre Schreibmotivation : " ohne Rücksprache mit der Familie» hätte Felix seine "Lebenserinnerungen der Druckerschwärze preisgegeben ${ }^{19}$. Das Buch sei voller Unwahrheiten : «Was mutet er mir nicht alles zu! Wie spricht er von der Familie!» (Olympia, 13). Unter dem «Banner der Wahrheit » rufe sie, Olympia, nun «Memoir wider Memoir!»(Olympia, 14). Sie belässt es bei einem Beispiel: So habe der Vater keineswegs Selbstmord begangen dieser sei von Mutter Krull, Olympia und dem Paten Schimmelpreester nur vorgetäuscht worden, in Wahrheit sei er « in den Armen von Julchen Semmelweis von der Städtischen Bühne» (Olympia, 17) gestorben. Olympia berichtet im Folgenden vornehmlich von ihrer Beziehung zu dem Hochstapler « Docteur Gorbakow », dessen betrügerische Geschäfte sie mit großem Geschick für ihn abwickelt.

Der zweite Teil entstammt dem Tagebuch der Tochter Marie-Anastasia. Im dritten Teil ersinnt Olympia den Plan, eine «Pension» - ein Edelbordell - einzurichten. Ihr eingeschobener Briefwechsel zeigt, wie sie es versteht, sich während dieser Planungen finanziell über Wasser zu halten; Hilfe erbittet sie von ihrem Bruder Felix. Der vierte Teil ist aus seiner Sicht in der Ich-Form geschrieben. Dem Hochstapler Krull, der sich nun Lord Chesterton nennt, gelingt es, den «Kanonenfabrikanten Krupp» zur Finanzierung des Unternehmens zu bewegen - über den Verwendungszweck seiner Spende wird er freilich getäuscht. Zudem erreicht er, dass Gorbakow seiner Schwester wertlosen Grundbesitz schenkt, den Krull schnell aufwerten kann: Er macht die Gesellschaft glauben, man habe einen Teil dem «herrlichen jungen Kaiser zwecks Errichtung einer Erholungsstätte» (Olympia, 333) für Offiziere geschenkt. Olympia ist saniert. Hatte er so für seine Schwester alles zum Besten wenden können, ereilt ihn selbst sein Schicksal: die Polizei war ihm gefolgt und verhaftet ihn wegen Juwelendiebstahls.

Der Roman enthält eine Fülle von Anspielungen ${ }^{20}$. Fünf Bezugsebenen lassen sich identifizieren, ohne dass die hier gewählte Reihenfolge eine Hierarchisierung bedeutete. Vier von ihnen stehen in Verbindung mit der Person oder dem Werk Thomas Manns. 

Fünfzigerjahren Neumanns Werke in einer Gesamtausgabe heraus. Zu diesem Zweck sollten der Roman Karriere (1931), die Hochstaplernovelle (1930) sowie Blinde Passagiere (1935) zu einem Band zusammengefasst werden. «Diesen dreien die Substanz eines Gesamtausgabenbandes zu geben", so Neumann, "bedurfte es mindestens eines vierten - und so entstand diese ,Olympia', in der ich meine alten Gestalten noch einmal [...] auftreten lassen wollte ${ }^{21} »$.

16 Eine dieser Gestalten ist Lord Chesterton, der es in der Hochstaplernovelle als genialer Schwindler zu großer Perfektion in Geschicklichkeit, Selbstkontrolle und souveräner Beherrschung seiner Umwelt gebracht hat. Das Hochstaplermotiv findet seinen pointierten Ausdruck im Falschspiel, das auch Thomas Manns Krull wohl noch lernen sollte $^{22}$, die Novelle ihren Höhepunkt in der wechselseitigen Entdeckung zweier Hochstapler. Bis auf kleinere, naheliegende motivische Übereinstimmungen Chesterton lässt sich von einem Museumsdirektor in die Kenntnisse der Antike einweihen, eine Szene, die an Professor Kuckuck erinnern kann - ist die Anlage der Novelle und der Bekenntnisse grundverschieden.

17 Neumann lässt nun Felix Krull in Olympia unter anderem als Lord Chesterton auftreten und macht ihn damit zu einer Weiterführung seiner Figur. Genauso wird Olympia zur Weiterführung einer Neumannschen Romangestalt, nämlich der Protagonisten des Romans Karriere. In ihm wird in der Ich-Form die Geschichte der ungarischen Animierdame Erna erzählt, die zunächst von einem Geschäftsmann aus einem billigen Tanzlokal « befreit » wird. Durch ihn lernt sie einen falschen Baron kennen, dann - sie macht « Karriere » - einen englischen Lord, der sie adoptiert. In Warschau fallen Erna die Geschäfte des Barons in die Hände. Es sind bis zur wörtlichen Übernahme von Namen und ganzen Passagen dieselben Transaktionen, die Olympia für den Hochstapler Gorbakow in Straßburg erledigt ${ }^{23}$. Olympia. Der zweite Teil, in dem Marie-Anastasia Geheimnisse aus ihrem Tagebuch preisgibt, ist eine zum Teil wörtliche Übernahme der ersten neun Kapitel des Romans Meine schöne $\mathrm{Mama}^{24}$. Neumann hatte ihn - was zu diesem Zeitpunkt nicht bekannt war - 1956 gemeinsam mit seiner Frau unter dem Pseudonym Mathilde Walewska verfasst.

Das mixtum compositum wird durch die durchgängige inhaltliche Ausrichtung auf die Haupt- und Titelfigur Olympia zusammengehalten. Auf der zweiten Bezugebene liegen die Anspielungen auf Handlung und Personenkonstellation der Bekenntnisse.

Bei Thomas Mann wird Olympia nur zu Beginn am Rande erwähnt. Die Schilderung durch ihren Bruder ist wenig schmeichelhaft: Olympia ist ein «ein dickes und außerordentlich fleischlich gesinntes Geschöpf ${ }^{25}$, das sich bei Abendgesellschaften im Hause Krull «einer geistlosen Völlerei» überlässt und «hinter gespreiztem Fächer » dumm «kichert» (GW VII, 278). Gefallsüchtig machen Olympia und ihre Mutter durch « Übertreibungen in der Form ihrer Hüte von sich reden » (GW VII, 279). Sie zeichnet sich durch «frauenhafte Unwissenheit» (GW VII, 334) aus und neigt zur «Bigotterie " (GW VII, 324). Alles in allem : Olympia ist dick, dumm und eitel - dass Leutnant Übel «in Liebe zu Olympia entbrannt " ist, kann Krull nur als «töricht " verstehen (GW VII, 316). Sie sollte später, so Krull, «nicht ohne Beifall die Operettenbühne [beschreiten] » (GW VII, 276). Die wenigen Andeutungen lassen sie als die Karikatur einer koketten Diva erscheinen, die ihre "Kunst », von einem windigen 
Geschäftsmann vermittelt, auf zweitrangigen Bühnen feilbietet und dafür «Beifall » bekommt ${ }^{26}$.

21 Neumann greift diese wenigen Aussagen zur Figur auf und lässt Olympia zur gescheiterten Wagnerinterpretin werden. Der parodistische Zug der Figur bei Thomas Mann wird grotesk gesteigert, Olympia ist vulgär und mondän, nur auf Äußerlichkeiten bedacht, eine Falschspielerin, unfähig zu ehrlichem Gefühl. Die Korruption durch das ausschließliche Leben im Schein gibt sie an ihre Tochter weiter : Wo sie sich vornimmt, in die «Erziehung » ihrer Tochter « einzugreifen », unterrichtet sie über den Gebrauch von Kosmetika (Olympia, 214).

22 Als weitere direkte Bezüge zu den Bekenntnissen sind daneben die oben erwähnten Aussagen zum Elternhaus sowie Olympias Zurückweisung der Beschreibung durch den Bruder zu nennen (Olympia, 27). Krulls Auftritt in Neumanns Roman fügt der Figur nichts Neues hinzu, abgesehen davon, dass Olympia eine Reihe von Namen aufzählen kann, unter denen er noch « tätig " gewesen sei (Olympia, 10) ${ }^{27}$.

Über diese Anspielungen hinausgehend parodiert Neumann auf einer dritten Ebene den Wahrheitsanspruch autobiographischen Schreibens. Olympia thematisiert ganz offen « die Elastizität der sogenannten Vergangenheit » (Olympia, 46f.). Marie-Anastasia steht ihr nicht nach, wenn sie glaubt, « daß man ohne Lügen vielleicht überhaupt nichts dem Papiere vermitteln kann » (Olympia, 236).

24 Neumann gibt mit der Parodierung der Autobiographie ein Beispiel dessen, was er selbst als "Vergröberung" der Parodie Thomas Manns betrachtete ${ }^{28}$, denn die Bekenntnisse sind bereits eine solche Parodie. Während Krull noch darauf beharrt, die Wahrheit zu sagen, wo er die Hochstapelei im Schreiben fortsetzt, ist hier die Desillusionierung des hochstaplerischen Schreibens perfekt.

Als Verweis auf eine autobiographische Bedeutungsebene lässt sich viertens die Schlusspointe interpretieren, in der sich Felix und Gorbakow als Hochstapler gegenüberstehen und gegenseitigen Respekt zollen. Auf ihr wird auch das Verhältnis Neumanns zu Thomas Mann kommentiert. Einerseits greift die Begegnung das Motiv der Hochstaplernovelle auf, andererseits, so Ulrich Scheck, handelt es sich um die " hintergründige Verbeugung des Neumannschen vor dem Mannschen Hochstapler ${ }^{29}$. Mehr noch : ebenso wie Thomas Mann im Hochstaplertum sein Künstlertum parodierte, hat Neumann das Schreiben als Hochstapelei bezeichnet ${ }^{30}$. Neumann, der « mit fremden Federn" schrieb, hat dieses Motiv so häufig literarisch verarbeitet, dass der Hochstapler als zentrale Figur seines Werkes betrachtet werden kann. Auch ihm ist der Hochstapler Selbstparodie, und so erweist in Olympia nicht nur sein Hochstapler dem Thomas Manns die Reverenz, sondern auch Neumann Thomas Mann; es ist die "schicksalskameradschaftliche Durchschauung " $^{31}$ - mit einer versteckten Spitze: Neumanns Gorbakow empfiehlt Manns Krull einen «'Leitfaden der Hochstapelei ! Aber' - so fügte er noch leichter hinzu - ,wem empfehle ich das ?'«(Olympia 320f.). Er durchschaut Krull zuerst, ist in der souveräneren Position, woraufhin Krull «zuckt» und kurzzeitig seine Sicherheit verliert. Neumann fiktionalisiert hier die Erfahrung, die er mit Thomas Mann gemacht haben will, dass man nämlich sein "Doppelspiel ", in dem man ihn auch als Parodist seiner selbst erkannt hatte, « submissest zu übersehen hatte $»^{32}$.

Neben diesen vier Bezugsebenen tritt schließlich fünftens - und hier ist nun die "Vertracktheit der Aggression » zu suchen - der offensichtliche Antisemitismus der Figuren Olympia und Felix, der als Thema durchgängig präsent ist ${ }^{33}$. Gedankenlos 
streut Olympia antisemitische Klischees in ihren Bericht ein ; "Sparsamkeit » (Olympia, 63) und «Sinnlichkeit fürs Geschäft » sind ihr selbstverständlich jüdische Eigenschaft. Sie spricht von einem "philosophisch veranlagte[n], wenn auch israelitische[n] Bekannte[n]» (Olympia, 19); ein anderer ist "ein feinsinniger Mann, wenn auch Israelit ». Olympia vermeidet hartnäckig das Wort «Jude», und ihr Antisemitismus steht immer kurz vor dem Umschlag in einen fragwürdig enthusiastischen Philosemitismus: Sie habe "eine Schwäche für unsere Volksgenossen jener faszinierenden Konfession! [...] Ah, was für ein Volk» (Olympia, 63) ${ }^{34}$. Sie, deren ganzes Weltbild sich aus Vorurteilen zusammensetzt, beteuert : «Vorurteil liegt mir fern!» (Olympia, 54). Dasselbe gilt für Felix, der gegenüber dem Juden Siebenschein äußert, " 'Ich kannte mal an der Universität einen Israeliten - aber hochanständigen Mann' », und sich mit seinen Lesern augenzwinkernd ins Einvernehmen setzt: "Aber nichts gegen die Israeliten - habe ich recht?» (Olympia, 304). Es finden sich neben diesen noch viele ähnliche Beispiele antisemitischer Klischees bei beiden, Felix und Olympia. In den Bekenntnissen finden sich zwei Stellen, an denen sich Felix Krull zu Juden äußert - beide besitzen einen antisemitischen Unterton ${ }^{35}$. Diese Einstellung des Erzählers Krull erscheint bei Thomas Mann jedoch kaum als ein ausreichend deutlicher Charakterzug der Figur, sodass eine Krull-Parodie darauf basieren könnte - das funktionierte nur, wenn das Parodierte hinreichend an das Original gemahnte. Es steht vielmehr $\mathrm{zu}$ vermuten, dass auf Thomas Mann selbst angespielt wird ${ }^{36}$. Offensichtlich wollte Neumann die sich wandelnden und zum Teil widersprüchlichen Äußerungen Thomas Manns zum Judentum kommentieren - und zwar auf «vertrackte» Weise, daher die Verquickung anti- und philosemitischer Aussagen. Es ist hier nicht der Ort, Äußerungen Thomas Manns und damit der möglichen Berechtigung der Neumannschen Kritik im Einzelnen nachzugehen. Nur so viel: Widersprüche - mit denen sich die Germanistik erst sehr viel später intensiver auseinander zu setzen begann - sind unleugbar; sie bestehen zwischen der unter dem Eindruck des nazistischen Antisemitismus unzweideutigen Parteinahme für das jüdische Volk einerseits und den Darstellungen jüdischer Figuren im Werk andererseits ${ }^{37}$. Letztere zeigen ein Oszillieren zwischen Faszination und Vorurteil, sind ambivalent, niemals vorurteilsfrei. Die Zählebigkeit von Stereotypen im Werk wird an den Bekenntnissen besonders greifbar: das antijüdische Klischee vom halsabschneiderischen Bankier, durch den Erzähler Krull geäußert, findet sich im Buch der Kindheit, entstammt mithin einer frühen Schaffensperiode. Es überlebt die Zeitenläufe mit der erneuten Publikation im Roman, der 1954 als Der Memoiren erster Teil erscheint, während ansonsten, wie Jacques Darmaun feststellt, die «Judenproblematik und symbolik nun [nach dem Doktor Faustus, H.P.] beinahe vollständig [verschwindet] ${ }^{38}$. $~ B e a c h t e t$ wurde das von der Kritik 1954 nicht. Neumann legt Widerspruch ein gegen das Zugleich von nichtfiktionaler projüdischer Stellungnahme und dem unterstellten Vorurteil im fiktionalen Werk, wenn Felix Krull in seinem Roman Olympia die Vorurteile seines Gesprächspartners Krupp bedient und dann den Leser anspricht : «Aber nichts gegen die Israeliten - habe ich recht?»

Neumann, selbst Jude, tat sich auch mit dezidiert projüdischen Äußerungen schwer, "philosemitische Sonderbehandlung» war ihm «nichts als Antisemitismus " ${ }^{39}$, er klagte zweierlei an : den "schwarzen und den weißen Antisemitismus $»^{40}$. So meinte er wohl auch Thomas Mann - aber : es handelt sich nicht um eine Abrechnung, nicht um ein abschließendes Urteil, sondern um eine "versteckte Attacke $»^{41}$ im Sinne einer Provokation, eines Anstoßes zu einer Debatte, die zu führen das Thomas Mann- 
Publikum noch nicht bereit war. Damit zielte Neumann nicht nur auf den Autor selbst, sondern genauso auf dessen Leser. Dass es ihm um diese Art von " Aufklärung » zu tun war, zeigt eine spätere Auseinandersetzung mit Erika Mann als der Sachwalterin des väterlichen Erbes : 1968 veröffentlichte Neumann in seinem Buch Vielleicht das Heitere einen Brief Thomas Manns aus dem Jahre 1920 an Hanns Johst, den späteren Präsidenten der Reichschrifttumskammer ${ }^{42}$. Thomas Mann lobt Johst darin, nichts « mit jener gallojüdischinternationalistischen ,Geistigkeit' zu schaffen zu haben », die das deutsche Geistesleben tyrannisiere ${ }^{43}$. "Diesen Brief ", so kommentierte Neumann, « muß einer kennen, der sonst in so vielem ja sagt zu Thomas Mann ${ }^{44}$.» Erika Mann habe ihn bei der Herausgabe der Briefe unterschlagen. Erika Mann hingegen behauptete, den Brief nicht gekannt zu haben, doch das glaubte ihr niemand so recht, denn er war bereits veröffentlicht worden ${ }^{45}$. Als Herausgeberin der Briefe ihres Vaters stand sie nicht gut da.

Zusammenfassend lässt sich feststellen, dass der Thomas Mann gewidmete Roman Olympia nicht ausschließlich auf dessen Bekenntnisse anspielt, sondern zunächst auf das eigene Werk Neumanns. Es soll nicht abschließend bewertet werden, wie sinnreich diese Anspielungen im einzelnen sind. Es bleibt jedoch beispielsweise zu fragen, ob etwa das ausführliche Selbstzitat aus Meine schöne Mama nicht vornehmlich arbeitsökonomische Gründe hatte.

- Von Interesse war hier vielmehr der Bezug zu Thomas Mann. Neumanns Roman ist keine Fortsetzung der Bekenntnisse, die die Figur Krull um irgendeinen Zug bereicherte ; auch der interpretative Ausbau der Olympia-Erwähnungen bei Thomas Mann zu einer eigenen Handlung bei Neumann eröffnet keine neue Perspektive auf den Roman.

31 Liest man ihn hingegen als eine Parodie auf die Bekenntnisse, die auf das bereits bei Thomas Mann parodistisch angelegte Hochstaplermotiv zielte, handelt es sich lediglich um eine "Vergröberung» der Parodie ${ }^{46}$. Die subtile Parodie wird ins Skurrile übersteigert. Auch die Parodierung des Wahrheitsanspruchs autobiographischen Schreibens zeigt keine parodistische Kritik an den Bekenntnissen, da diese ihn selbst schon infrage stellen. Olympia bleibt auf diesen Bezugsebenen eine affirmativ arrangierte Adaption.

Möglich bleibt, dass der Parodist Neumann hier für einmal eine Parodie zur Grundlage einer Parodie machte - was seiner eigenen Ästhetik der Parodie zufolge einem Nullsummenspiel gleichkommt - und somit auf das eigene « Handwerk » zielte ; die Selbstdarstellung des Schriftstellers (der in seinem Fall ein Parodist ist) als Hochstapler deutet darauf hin.

Was die beiden letztgenannten Bezugsebenen, die Anspielungen auf Neumanns Beziehung zu Thomas Mann und den Anti- und Philosemitismus, anbelangt, sind die zu den Bekenntnissen hergestellten Beziehungen Mittel zum Zweck: Sie werden in der über das einzelne Werk hinausweisenden Auseinandersetzung eines Autors mit einem anderen instrumentalisiert, um auf die Identität des letzteren zu verweisen. In Neumanns Parodieverständnis besitzt diese Form instrumentalkritischer Parodie, die nicht auf Form oder Motive des Primärwerks sondern auf seinen Urheber zielt, einen 
zentralen Stellenwert: "Parodie ist Nachahmung mit Polemik gegen den Nachgeahmten » - nicht unbedingt gegen das Nachgeahmte ${ }^{47}$.

Gerade die Anti- und Philosemitismus-Thematik wurde von der zeitgenössischen Kritik hartnäckig übersehen - damit bestätigte sich Neumanns in Literatur verpackter Vorwurf in der Realität: die Unwilligkeit, über das Thema zu sprechen. Auch Erika Mann hat sich dazu in ihren zahlreichen Äußerungen zu Olympia nicht geäußert. Man beschäftigte sich vielmehr aufgeregt mit der durch den Plagiatsvorwurf der MannErben und des S. Fischer Verlags aufgeworfenen Frage, ob es sich nicht um eine unzulässige Bearbeitung handelte ${ }^{48}$. Diese Debatte gab Neumann erst recht die Gelegenheit, gegen die in seinen Augen von Erika Mann betriebene kritiklose Kanonisierung Thomas Manns zum «Klassiker» Einspruch zu erheben. Ihrer Entrüstung über das Buch begegnete er nicht ohne Ironie, er « beneide Thomas Manns Schatten um die wachsame Tüchtigkeit seiner Erbengemeinschaft ${ }^{49}$." Erika Mann unterdessen wurde nicht müde, auf das künstlerische Gefälle zwischen den Romanen hinzuweisen. Je mehr sich abzeichnete, dass der Klage nicht stattgegeben werden würde - dies hätte einen neuen Plagiatstatbestand geschaffen, das Gericht drängte auf einen Vergleich - desto mehr scheint es, als sei auch ihr die Auseinandersetzung um die Bekenntnisse Mittel zum Zweck geworden, als sollte mit der öffentlichen Herabsetzung der Olympia und dem Zweifel an der "Zurechnungsfähigkeit des Autors » ${ }^{50}$ ein Beispiel gegeben werden : Nicht umsonst wird auf Thomas Manns Roman in der Klageschrift meist nur als "der Meisterroman » Bezug genommen ${ }^{51}$. An einer solchen Qualifizierung war nicht zu rütteln, das war auch nicht zu parodieren, Neumann leide schlicht unter einem "pathologischen Thomas Mann-Komplex $»^{52}$, die Widmung des Buches sei eine «literarische Perversität " ${ }^{33}$. Darauf konnte Neumann nur noch kontern : « die Förster-Nietzsche [...] hat in ähnlicher Weise angegeben ${ }^{54}$. »

Die unfruchtbare einjährige Auseinandersetzung um die Daseinsberechtigung des Neumannschen Hochstapler-Romans endete im März 1962 mit einem Vergleich, nachdem Neumann ganze 19 Zeilen geändert hatte, nicht ohne dass auch diese Änderungen nochmals eine Spitze enthielten: sie sind selbst hintergründige Kommentare zum angedrohten Prozess. Hochstapelei allenth alben.

\section{NOTES}

1. Die hier präsentierten Ergebnisse sind Teil derzeitiger Vorarbeiten zu meiner Dissertation über die Rezeptionsgeschichte der Bekenntnisse des Hochstaplers Felix Krull.

2. Hermann Kesten : "Eine gelungene Parodie auf literarische Parodien und Parodisten ", in : Die Kultur, München, 26.05.1961.

3. Marcel Reich-Ranicki : Deutsche Literatur in Ost und West, München, Piper, 1963, S. 251.

4. Thomas Mann an Robert Neumann, 07.10.1927. Eine Abschrift des Briefes befindet sich in der Dokumentationsstelle des österreichischen Widerstands, Wien, Akt. Nr. 11548/3. Zitiert nach Ulrich Scheck : Die Prosa Robert Neumanns. Mit einem bibliographischen Anhang, New York u.a., Lang, 1985. (American University Studies. Series I, 43), S. 33. 
5. Kurt Desch an Erika Mann, 31.01.1961. Stadtbibliothek München. Monacensia Literaturarchiv. Nachlass Erika Mann. Nr. 231.

6. [Desch Verlag: Werbetext Olympia]. SULB Düsseldorf. Thomas-Mann-Sammlung, Signatur J III 1.

7. Robert Neumann : Ein leichtes Leben. Bericht über mich selbst und Zeitgenossen, Wien / München / Basel, Desch, 1963, S. 487.

8. Ebd. S. 404.

9. Ebd., 405.

10. In einem Interview mit Horst Bienek äußerte Neumann sich dazu: «Ich halte sie [die Parodien] für halb so wichtig, wie manche Leute glauben.» Und nach den Gründen für die wechselnden Stile seiner nichtparodistischen Werke gefragt: "Weil ich mich in keinem dieser parodierten Stile wohlgefühlt und nach dem eigenen Stil gesucht habe, und vielleicht mehr experimentiert, als andere Autoren. Diese Experimentiersucht macht einen [...] unpopulär. [...] Ich finde, daß im Experiment ein großes Abenteuer liegt, ich finde, daß ich den Versuch, einen eigenen Stil zu finden, nicht ganz erfolglos angestellt habe, aber es kann natürlich sein, daß ich unrecht habe. » Horst Bienek : Werkstattgespräche mit Schriftstellern, München, Hanser, 1962, S. 59 . 11. Vgl. z. B : «In dem englisch geschriebenen Roman [Scene in passing, 1942] von Robert Neumann. » (28.6.1943). Vgl. auch die Einträge : «Bücher : Robert Neumann, eine Novelle [Blinde Passagiere]» (02.05.35) und «Robert Neumann schickt aus Oxford sein neues Buch» (28.06.1946). Thomas Mann : Tagebücher, hrsg. von Peter de Mendelssohn und Inge Jens, Frankfurt/Main, S. Fischer, 1976-1995.

12. Die Tagebücher Thomas Manns wissen darüber nicht mehr zu berichten als : «Besuch von Neumann » und «Zum Abendessen Robert Neumann » (17.09.1935; 06.04.1936). Über Gespräche allgemeiner Natur hielt Thomas Mann nicht mehr fest als z.B. «Deutschland und Literatur » (10.07.1947). Thomas Mann, Tagebücher, a.a.O.

13. Thomas Mann an Robert Neumann, 28.03.1955, zitiert nach : Robert Neumann [et al.] : Ein Skandal, der keiner war. Der Fall Olympia und der Fall Mathilde Walewska - Die Dokumente, in : Die Welt, 28.10.1961. Auszugsweise wiedergegeben in Anmerkung 2 zum Tagebucheintrag vom 26.03.55. Thomas Mann : Tagebücher, a.a.O.

14. Robert Neumann an Thomas Mann, 02.04 .1955 , zitiert nach : Robert Neumann [et. al] : Ein Skandal, der keiner war, a.a.O.

15. Robert Neumann : Ein leichtes Leben, a.a.O., S. 385. Dort auch die folgenden Zitate.

16. Robert Neumann: Vielleicht das Heitere. Tagebuch aus einem anderen Jahr, Wien / München / Basel, Desch 1968, S. 188.

17. Rudolf Walter Leonhardt : «Vielleicht das Persönliche », in : Robert Neumann : Typisch Robert Neumann. Eine Auswahl, hrsg. von Helga Heller-Neumann, Wien / München / Basel, Desch 1975, S. 7-12, hier S. 11.

18. Robert Neumann : Vielleicht das Heitere, a.a.O., S. 591.

19. Robert Neumann : Olympia, Wien / München / Basel, Desch, 1961, S. 10. Alle Seitenangabe beziehen sich auf die erste Fassung des Romans. Im Folgenden im Text zitiert als Olympia, [Seite]. 20. Vgl. auch Ulrich Scheck, a.a.O., S. 92-101.

21. Robert Neumann an Kurt Desch, 03.11.1960, zitiert nach: Robert Neumann [et al.], Ein Skandal, der keiner war, a.a.O. Vgl. auch die Aussage im Interview mit Horst Bienek: «Und ,Olympia' habe ich das Buch nach einer Figur genannt, die Mann im ,Krull' am Rande erwähnt und nicht ausgeführt hat. Sie ist de facto eine Weiterführung der Erna in meinem Roman ,Karriere', und ihre Tochter ist Weiterführung wieder einer anderen Gestalt, und Lord Chesterton heißt schon ,der Held' meiner Hochstaplernovelle aus dem Jahr 1930. « Horst Bienek, a.a.O., S. 63. 22. Vgl. Thomas Manns Notizen zu den Bekenntnissen, abgedruckt in der Dokumentation bei Hans Wysling: Narzißmus und illusionäre Existenzform. Zu den 'Bekenntnissen des Hochstaplers Felix Krull', Bern / München, Francke, 1982. (Thomas-Mann-Studien 5), S. 389-544, hier S. $465 f$. 
23. Vgl. Robert Neumann : Karriere, Wien / München / Basel, Desch, 1953, S. 67-97, und Robert Neumann, Olympia, a.a.O., S. 92-108.

24. Mathilde Walewska [ = Robert Neumann / Evelyn Mathilde Walewska Neumann] : Meine schöne Mama, Wien / München / Basel, Desch, 1956.

25. Thomas Mann : Bekenntnisse des Hochstaplers Felix Krull. Der Memoiren erster Teil, Gesammelte Werke in 13 Bänden, Bd. VII, Frankfurt/Main, S. Fischer, 1974, S. 276. Im Folgenden im Text zitiert als GW VII, [Seite].

26. Auch Olympia ist somit eine Parodie eines dekadenten, » wirkungssüchtigen « Künstlertums ; die Anspielungen auf ihre »Kunst « sind ein thematisches Seitenstück zum Müller-RoséKomplex.

27. Als weitere indirekte Anspielung ist die Annäherung Gorbakows an Marie-Anastasia zu sehen. Gorbakow begehrt damit in Mutter und Tochter ein « Doppelbild», so wie Felix Krull Maria Pia und Zouzou begehrt. In beiden Fällen interveniert die Mutter, um die Tochter « aus dem Felde zu schlagen ».

28. Vgl. Robert Neumann an Thomas Mann, 02.04.1955, a.a.O.

29. Ulrich Scheck, a.a.O., S. 96.

30. Vgl. Robert Neumann : Ein leichtes Leben, a.a.O., S. 405.

31. Ebd., S. 385.

32. Ebd.

33. Vgl. Andrea Kriegner: Das Judentum im Romanwerk Robert Neumanns, masch. Diplomarbeit, Universität Innsbruck, 1992, S. 98f. ; 130-132.

34. Vgl. auch S. 31 : « mit dem für diese faszinierende Konfession so typisch genialen Erfassen des falschen Augenblicks!.

35. Der im Hause Krull verkehrende jüdische Bankier ist «einer der verhärtetsten Halsabschneider [...], welche jemals bedrängte und unbedachte Geschäftsleute in ihre Netze gelockt haben » (319), und wird indirekt von Krull für den Konkurs mitverantwortlich gemacht. Das von Krull in Frankfurt sehnsüchtig bewunderte Zwillingspaar ist möglicherweise jüdisch, wie Krull vermutet: " - ich möchte mich nicht verbürgen und ließe mich dadurch in meiner Schwärmerei nicht beirren, denn luxuriös erzogene Kinder dieses Stammes können höchst anziehend sein » (345).

36. Vgl. Ulrich Scheck, a.a.O., S. 96.

37. Vgl. z. B. Jacques Darmaun: Thomas Mann, Deutschland und die Juden, Tübingen, Niemeyer, 2003. (Conditio Judaica 40), z.B. S. 2 u.ö. Zum Folgenden ebd., 293 f.

38. Ebd., S. 293.

39. Robert Neumann : Ein leichtes Leben, a.a.O., S. 534.

40. Ebd., S. 535.

41. Ulrich Scheck, a.a.O., S. 96.

42. Robert Neumann : Vielleicht das Heitere, a.a.O., S. 592f. Vgl. dazu Irmela von der Lühe : Erika Mann. Eine Biographie, Frankfurt/Main / New York, Campe, 1993. (Geschichte und Geschlechter. Sonderband), S. 265.

43. Thomas Mann an Hanns Johst, 16.09.1920. Die Briefe Thomas Manns. Regesten und Register, hrsg. von Hans Bürgin und Hans-Otto Mayer, fortgeführt von Gert Heine und Yvonne Schmidlin, 5 Bände, Frankfurt/Main, S. Fischer, 1976, Bd. 1, Nr. 20/92.

44. Robert Neumann: Vielleicht das Heitere, a.a.O., S. 593.

45. Nach von der Lühe, a.a.O., S. 266 in : Kurt Ziesel : Das verlorene Gewissen. Hinter den Kulissen der Presse, der Literatur und ihrer Machtträger von heute, München, Lehmann, 1957, S. 197f. In der zweiten Auflage 1958 nicht enthalten.

46. Vgl. Robert Neumann an Thomas Mann, 02.04.1955, a.a.O.

47. Zitiert nach Wolfgang Karrer: Parodie, Travestie, Pastiche, München, Fink, 1977, S. 37. Karrer identifiziert diese Art von Kritik als zentrales Motiv Neumanns. Dies bedeutet jedoch nicht, wie 
Karrer meint, dass Neumann sich in dieser Kritik, die auf den Autor hinter dem Werk zielt, auf lebende Zeitgenossen beschränken musste.

48. $\mathrm{Zu}$ dem Prozess vgl. auch von der Lühe, a.a.O., S. 266-268, und Hansgeorg Blechschmid: Thomas Mann und das Recht, München 2004. (Thomas-Mann-Schriftenteihe 3), S.144 j.

49. Robert Neumann an Erika Mann, 22.02.1961. Monacensia Literaturarchiv, a.a.O.

50. Erika Mann : « Mit eigener Feder », in : Das Schönste 1961, Jg. 7, Nr. 7, S. 32f., hier S. 32.

51. Klage des S. Fischer Verlags und Katharina Manns gegen den Verlag Kurt Desch und Robert Neumann, eingereicht beim Landgericht Stuttgart, 15.03.1961 (Durchschrift ; 30 S.). Monacensia Literaturarchiv, a.a.O. : « Meisterroman » : S. 7, 9, 17, 19, 22, 23, 25 ; « Meisterwerk » : S. 10, 11.

52. [Erika Mann] : «Thomas Mann und die ,Schöne Mama'. Ein Streitgespräch mit Erika Mann», in : Gießener Anzeiger, 07.10.1961.

53. Erika Mann: «Ein Skandal, der einer ist'. Eine Entgegnung von Erika Mann auf Robert Neumann », in : Die Welt, 04.11.1961.

54. Robert Neumann : «Die Mathilde Walewska-Story », in : Das Schönste 1961, Jg. 7, Nr. 10.

\section{RÉSUMÉS}

Der Beitrag entschlüsselt den Anspielungsreichtum des Romans Olympia (1961) in seinen Hintergründen. Robert Neumann greift in ihm ein zentrales Motiv seines eigenen Werkes auf : Die Hochstapelei. Darüber hinaus ist der Thomas Mann gewidmete Roman eine Parodie auf die Bekenntnisse des Hochstaplers Felix Krull, gegen den die Erben Thomas Manns einen Plagiatsprozess anstrengten. In Olympia tritt Felix Krull selbst auf, die Titelheldin ist als seine Schwester ebenfalls dem Roman Thomas Manns entlehnt. Schließlich fiktionalisiert Neumann in der Begegnung «Felix Krulls» mit einer eigenen Hochstapler-Figur seine problematische Beziehung zu Thomas Mann und kommentiert mit den anti- und philosemitischen Äußerungen der entlehnten Gestalten das Verhältnis Thomas Manns zum Judentum.

Cet article met en lumière la richesse des allusions du roman Olympia (1961). Robert Neumann y aborde une problématique-clé de son œuvre : l'imposture. Ce roman, dédié à Thomas Mann et contre lequel ses héritiers ont intenté un procès pour plagiat, est une parodie de l'ouvrage. Felix Krull est un des personnages d'Olympia. Sa sœur, l'héroïne éponyme, est également empruntée au roman de Thomas Mann. Enfin, dans la rencontre de «Felix Krull» avec une autre figure d'imposteur, Neumann transcrit sa relation problématique avec Thomas Mann et fait allusion, à travers les propos antisémites et philosémites de ses personnages, à la relation de Thomas Mann au judaïsme.

\section{AUTEUR}

\section{HOLGER PILS}

Universität Heidelberg 